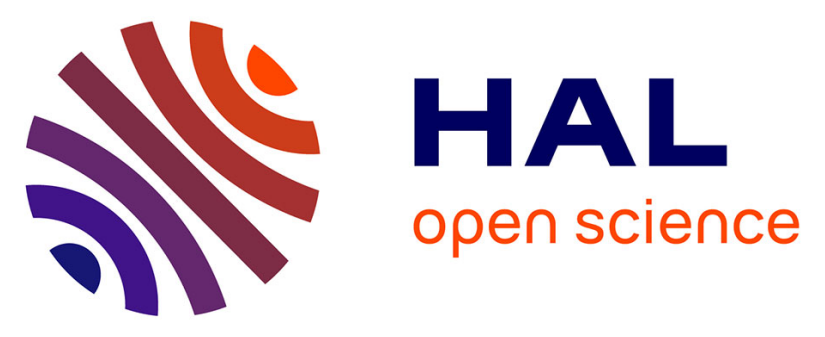

\title{
Padeliporfin vascular-targeted photodynamic therapy versus active surveillance in men with low-risk prostate cancer (CLIN1001 PCM301): an open-label, phase 3, randomised controlled trial
}

Abdel-Rahmène Azzouzi, Sébastien Vincendeau, Eric Barret, Antony Cicco, François Kleinclauss, Henk G. van Der Poel, Christian G. Stief, Jens Rassweiler, Georg Salomon, Eduardo Solsona, et al.

\section{- To cite this version:}

Abdel-Rahmène Azzouzi, Sébastien Vincendeau, Eric Barret, Antony Cicco, François Kleinclauss, et al. Padeliporfin vascular-targeted photodynamic therapy versus active surveillance in men with lowrisk prostate cancer (CLIN1001 PCM301): an open-label, phase 3, randomised controlled trial. Lancet Oncology, 2017, 18 (2), pp.181-191. 10.1016/S1470-2045(16)30661-1 . hal-01484971

HAL Id: hal-01484971

https://hal-univ-rennes1.archives-ouvertes.fr/hal-01484971

Submitted on 21 Jun 2018

HAL is a multi-disciplinary open access archive for the deposit and dissemination of scientific research documents, whether they are published or not. The documents may come from teaching and research institutions in France or abroad, or from public or private research centers.
L'archive ouverte pluridisciplinaire HAL, est destinée au dépôt et à la diffusion de documents scientifiques de niveau recherche, publiés ou non, émanant des établissements d'enseignement et de recherche français ou étrangers, des laboratoires publics ou privés. 
1 TITLE: Padeliporfin Vascular-targeted Photodynamic Therapy Versus Active Surveillance:

2 A Randomised Clinical Trial in Men with Low-risk Prostate Cancer

3 RUNNING TITLE: Photodynamic Therapy for Low-risk Prostate Cancer

\section{AUTHORS AND AFFILIATIONS:}

5 Abdel-Rahmène Azzouzi, M.D. ${ }^{1}$, Sébastien Vincendeau, M.D. ${ }^{2}$, Eric Barret, M.D. ${ }^{3}$, Antony

6 Cicco, M.D. ${ }^{4}$, François Kleinclauss, M.D. ${ }^{5}$, Henk G. van der Poel, M.D. ${ }^{6}$, Christian G. Stief,

7 M.D. ${ }^{7}$, Jens Rassweiler, M.D. ${ }^{8}$, Georg Salomon, M.D. ${ }^{9}$, Eduardo Solsona, M.D. ${ }^{10}$, Antonio

8 Alcaraz, M.D. ${ }^{11}$, Teuvo T. Tammela, M.D. ${ }^{12}$, Derek J. Rosario, M.D. ${ }^{13}$, Francisco Gomez-

9 Veiga, M.D. ${ }^{14}$, Göran Ahlgren, M.D. ${ }^{15}$, Fawzi Benzaghou, M.D. ${ }^{16}$, Bertrand Gaillac, M.D. ${ }^{16}$,

10 Billy Amzal, Ph.D. ${ }^{17}$, Frans M.J. Debruyne, M.D. ${ }^{18}$, Gaëlle Fromont, M.D. ${ }^{19}$, Christian

11 Gratzke, M.D. ${ }^{7}$, Mark Emberton, FMedSci ${ }^{20}$ on behalf of the PCM301 Study Group

$12{ }^{1}$ Department of Urology, Angers University Hospital, Angers, France

$13{ }^{2}$ Department of Urology, CIC-INSERM 1414, Rennes University Hospital, Rennes, France

$14{ }^{3}$ Department of Urology, Institut Montsouris, Université Paris Descartes, Paris, France

${ }^{4}$ Department of Urology, Centre Catalan Urologie Andrologie, Cabestany, France

${ }^{5}$ Department of Urology, Besançon University Hospital, Besançon, France

${ }^{6}$ Department of Urology, Netherlands Cancer Institute - Antoni van Leeuwenhoek Hospital, Amsterdam, The Netherlands

${ }^{7}$ Department of Urology, LMU-Klinikum der Universität München, Munich, Germany 
${ }^{9}$ Martini-Clinic Prostate Cancer Center, University Medical Center Hamburg-Eppendorf,

${ }^{10}$ Department of Urology, Fundación Instituto Valenciano de Oncología, Valencia, Spain

${ }^{11}$ Department of Urology, Hospital Clinic, Barcelona, Spain

${ }^{12}$ Department of Urology, Tampere University Hospital and School of Medicine, Tampere,

Finland

${ }^{13}$ Academic Department of Urology, University of Sheffield, Royal Hallamshire Hospital, Sheffield, UK

${ }^{14}$ Department of Urology, Salamanca University Hospital GITUR-IBSAL, Salamanca, Spain and Department of Complex University Hospital, La Coruna, Spain

${ }^{15}$ Department of Urology, Skäne University Hospital, Malmö, Sweden

${ }^{16}$ Medical Department, STEBA Biotech, Paris, France

${ }^{17}$ LA-SER Analytica, London, UK

${ }^{18}$ Andros Men's Health Institute, Arnhem, The Netherlands

${ }^{19}$ Department of Pathology, Tours University Hospital, Tours, France

${ }^{20}$ Division of Surgery and Interventional Science, University College London, London, UK

CORRESPONDING AUTHOR: Prof. Mark Emberton

Address: UCL Faculty of Medical Sciences, 1A Maple House, 149 Tottenham Court Road,

40 Phone: +44 (0)203 1082105

$41 \quad$ Email: m.emberton@ucl.ac.uk 


\section{SUMMARY}

43

44

45

46

47

48

49

\section{Background}

Vascular-targeted photodynamic therapy (VTP), a novel tissue-preserving treatment for lowrisk prostate cancer (PC), has shown favorable safety and efficacy results in single-arm Phase I and II studies. This report presents results of a randomised, controlled, parallel-group clinical trial of padeliporfin VTP versus the standard of care, active surveillance (AS).

\section{Methods}

Men with low-risk, localised PC (no Gleason patterns 4 or 5) and no previous treatment were recruited from March 8, 2011 to April 30, 2013 at 47 European university centres and community hospitals. They were randomised (stratification by centre using balanced blocks) to VTP or AS. VTP consisted of $4 \mathrm{mg} / \mathrm{kg}$ padeliporfin administered intravenously over ten minutes via optical fibres inserted into the prostate to cover the desired treatment zone and subsequent activation by laser light for 22 minutes and 15 seconds. Both groups were followed-up for 24 months in accordance with best AS practice at the time of study design, i.e., biopsy at 12-month intervals and prostate-specific antigen measurement and digital rectal exam at 3-month intervals. The prespecified co-primary efficacy endpoints were histological progression of cancer and absence of any histology result definitively positive for cancer at Month 24. Treatment was open-label, but primary efficacy outcomes were evaluated in a blinded manner.

\section{Findings}

Of the 206 subjects randomised to VTP, 196 received treatment. At completion of the trial, all 413 randomised subjects (intention-to-treat population) were analysed for efficacy. VTP doubled time to progression (from 14. 1 [95\% CI: 12.9 to 23.8 ] months to $28 \cdot 3$ [95\% CI: 26.0 
to 30.6] months; $\mathrm{p}<0 \cdot 0001)$ and reduced the progression rate to approximately one-third that of AS (adjusted hazard ratio $=0 \cdot 34 ; 95 \%$ CI: $0 \cdot 24,0 \cdot 46 ; \mathrm{p}<0 \cdot 0001)$. VTP increased the probability of a negative prostate biopsy at 24 months post-treatment from 13.5\% (28 of 207 subjects) to $49 \cdot 0 \%$ (101 of 206 subjects) (adjusted risk ratio: $3 \cdot 67 ; 95 \%$ CI: $2 \cdot 53,5 \cdot 33$ ); $\mathrm{p}<0 \cdot 0001)$.

VTP was well tolerated. Genitourinary function showed transient deterioration in the VTP group, but no significant effects were seen at Month 24. The most common AEs in the VTP group were urinary tract infections (21 subjects) and AEs in the renal and urinary disorders (133 subjects) and reproductive system and breast disorders system organ classes (121 subjects). The most common serious side effect was retention of urine. Typically this event occurred on the first attempt to withdraw the urinary catheter (day-1 post-op). This was managed with immediate re-catheterization. The timing of a second attempt at removal of the urinary catheter was left to the discretion of the local investigator. This event occurred in 15 subjects, was severe in 3 subjects, and resolved within two months in all cases.

\section{Interpretation}

Padeliporfin VTP is a safe effective treatment for low-risk, localised PC that reduces the rate of histological progression compared to AS. It may allow more men to consider a tissuepreserving approach and defer or avoid radical therapy.

\section{$83 \quad$ Funding}

STEBA Biotech S.A.

\section{Registration}




\section{RESEARCH IN CONTEXT}

89

90

91

92

93

94

95

96

97

98

\section{Evidence before this study}

The idea of modifying our therapeutic target from the host organ to the tumour plus a margin has been the mainstay of surgical oncology during the latter half of the $20^{\text {th }}$ century. The principle is probably best exemplified in breast cancer, for which the previous standard of care, the Halsted radical mastectomy, has, with time and accumulating evidence, largely been replaced by breast preservation achieved by local excision with or without radiotherapy. We have seen the same process in renal cancer. Radical nephrectomy is currently performed only when partial nephrectomy and nephron preservation is neither practical nor possible. Ten years ago it was performed in all patients. The principles behind this transition are equivalence in terms of cancer-related outcomes but better function, greater patient acceptability, quicker recovery, and enhanced survivorship. Prostate cancer (PC) is the only solid organ cancer left for which this principle is not generally applied. Over the last decade several proof-of-concept studies of focal therapy for PC have been published, but they have typically been single-centre, small, and of relatively low quality. Having said this, these studies demonstrated the feasibility of more targeted treatment for PC and more importantly suggested high levels of patient acceptability because of excellent functional outcomes. More recently we have seen registered prospective development studies and formal Phase I and Phase II studies that demonstrate both safety and early (short-term) oncological efficacy. These studies have been summarised in Valerio's systematic review.

\section{Added value of this study}


Valerio's systematic review identified the need for comparative studies. To our knowledge, ours is the first such study. Because vascular-targeted photodynamic therapy (VTP) is an intervention involving both a drug (in this case, padeliporfin) and a device (laser light introduced into the prostate), it was subject to regulatory approval as a drug through the European Medicines Agency (EMA). A pivotal comparative study was thus necessary but was challenging to design in a manner that would be acceptable to both patients and clinicians and in which the same primary outcome could be assessed for both VTP and the comparator. We had three options for the comparator: surgery, radiotherapy, or active surveillance (AS). The first two were problematic in arriving at a primary outcome that could be applied to both the experimental arm and the control. Surgery (radical prostatectomy) would not be suitable for a biopsy-based outcome because there would be no prostate to biopsy. Radiotherapy, on the other hand, would be amenable to a protocol-required biopsy, but the histological outcome would be confounded by the necessary neoadjuvant and adjuvant androgen suppression that comprises the standard of care. Therefore, AS was the only comparator that could reasonably be employed over the intended time frame of the study. The task for the EMA and the PCM301 Study Group was to determine the upper and lower risk thresholds of this low-risk group that would define the upper and lower bounds of the study entry criteria. These criteria had the effect of excluding, within the limits of precision of the diagnostic methods available to us at the time, men that were at very low risk and therefore unlikely to progress and men that were at higher risk and therefore unlikely to be offered or indeed consent to AS.

These thresholds of low risk were in keeping with standard practice at the time of study design. Recent publications from Scandinavia and Canada on mature AS populations have subsequently shown that men towards the upper threshold of low-risk PC do fare worse in progression than men with very-low-risk disease. Modern diagnostic methods, including 
magnetic resonance imaging, allow us to identify these risk groups with considerably greater precision today than was possible at the time when the study was being considered by the EMA. Our results show that men with localised, low-risk PC can be treated in a way that not only preserves their genitourinary function but also results in a lower progression rate, greater chance of being declared disease-free, and reduction in need for whole-gland radical therapy in the form of surgery or radiotherapy.

\section{Implications of all the available evidence}

When this study was designed, our risk stratification methods at diagnosis were poor. The correction that was applied to mitigate the consequences of this imprecision was to offer radical therapy to nearly all men, irrespective of attributed risk. Today we attribute risk with greater precision using risk calculators, biomarkers, and imaging. Our study adds considerable weight to the argument that we need to move away from a one-size-fits-all approach to treatment and gradually replace it with a more risk-stratified approach to care.

We have AS for men at very low risk. We have radical therapy and multimodality treatments for men at high risk for whom the consequences of treatment are matched by benefit.

Between these two extremes, we now have VTP, an intervention that preserves prostate tissue when it is both possible and practical to do so. Given the precision of today's risk stratification, future research will need to explore both the patient preferences and the upper threshold of risk (as defined by tumour grade, volume, location, multiplicity) that should determine where the transition point exists where tissue preservation is likely to confer diminishing returns and should be supplanted by whole-gland radical therapy.

\section{INTRODUCTION}


157 Active surveillance (AS), a policy of delayed selective intervention, is an appropriate therapeutic option for low-risk prostate cancer (PC) that helps to mitigate the consequences of overtreatment. ${ }^{1}$ Most studies — though admittedly single-centre and noncomparative — have demonstrated favorable outcomes, but AS has been associated with fairly high intervention rates especially in cohorts with less stringent eligibility criteria. ${ }^{2}$ Intervention, or crossover to radical treatment (surgery or radiotherapy) or systemic therapy (androgen suppression), tends to be driven by — in descending order of frequency — pathological upgrading on repeat biopsy, biochemical progression, and patient choice. ${ }^{3}$

Focal therapy and AS are both tissue-preserving strategies. They share the goal of preserving prostate tissue and consequently function by delaying or avoiding radical whole-gland treatment in men in whom it is safe to do so. ${ }^{4}$ However, focal therapy differs from AS in that it treats disease - by the process of selective tissue ablation — above a certain risk threshold and monitors disease below that threshold, as the latter is deemed to be clinically insignificant. A risk-stratified clinical pathway that offers men focal therapy in a manner complementary to AS might result in two potential benefits: a reduction in the probability of failure or crossover to radical therapy and an increase in the proportion of men eligible and willing to undergo a tissue-preserving treatment.

Neither focal therapy nor AS has previously been assessed in a prospective, comparative efficacy study. Both have been assessed only in single-centre series, ${ }^{2,5,6}$ in which the outcomes were dependent on the population studied, the diagnostic precision at baseline, the intensity and manner of the reclassification tests, and the study duration. These limitations challenge informed decision-making by the patient because the attributes that are most likely to influence treatment selection are the failure rates and toxicity profiles of the two approaches and the likelihood of avoiding radical therapy. We present the results of what is to our knowledge both the first prospective comparative evaluation of the efficacy and safety of 
focal therapy and the first evaluation of AS in a comparative setting-rather surprisingly given that it is a recommended standard of care. The selective ablation in our focal therapy arm was achieved using vascular-targeted photodynamic therapy (VTP) with padeliporfin, an agent that achieves its tissue effects nonthermally and had previously been evaluated in both preclinical and clinical settings. ${ }^{7,8}$

\section{METHODS}

\section{Study design and participants}

Study CLIN1001 PCM301 was a randomised, controlled, parallel-group clinical trial of padeliporfin VTP versus AS for treatment of low-risk, localised PC. Men aged $\geq 18$ years with low-risk, localised PC diagnosed by transrectal ultrasound (TRUS)-guided biopsy and no previous treatment were enrolled, provided they were eligible to be exposed to a photosensitising agent and had no contraindications to undergoing magnetic resonance imaging (MRI). Participants were required to have low-risk PC but not very-low-risk PC. Men were eligible if one core of cancer that was free of Gleason patterns 4 or 5 was present provided that the cancer core length was between 3 and $5 \mathrm{~mm}$. In other words, if only one core was positive, only Gleason pattern 3 was permitted but in order to qualify the cancer core length had to be greater than or equal to $3 \mathrm{~mm}$ and less than or equal to $5 \mathrm{~mm}$. Men with 2 or 3 cores positive were also permitted, but cancer core length could not exceed $5 \mathrm{~mm}$. Clinical stage was limited to $\leq \mathrm{T} 2 \mathrm{a}$ (pathological or radiological up to $\mathrm{T} 2 \mathrm{c}$ disease permitted), prostate-specific antigen (PSA) $\leq 10 \mathrm{ng} / \mathrm{mL}$, and prostate volume $\geq 25$ and $<70 \mathrm{cc}$ ). These criteria were based on a study of prediction determinants prediction in AS subsequently reported by Welty et al. ${ }^{9}$ The performance status of the subjects was not a criterion for study inclusion. Instead, two overarching requirements had to be satisfied: men had to have a predicted life expectancy of 10 years or more and, in addition, had to be free of any medical 
conditions that were deemed to be a contraindication to general anaesthesia. Men with a

207

208

209

210

212

213

214

215

216

217

218

219

220

221

222

223

224

225

226

227

contraindication to MRI (e.g. cardiac pacemaker), factors excluding accurate reading of pelvic MRI (e.g. bilateral hip replacements), or any condition or history of illness or surgery that may have posed an additional risk to men undergoing VTP procedure were excluded. Criteria for subject removal from the study were occurrence of a serious adverse event (SAE) if recommended by the investigator, subject withdrawal, or a major protocol violation.

The study was conducted in compliance with Good Clinical Practice and according to a written protocol approved by each centre's ethics committee. All subjects provided written informed consent. The trial was completed in accordance with the protocol.

\section{Randomisation and masking}

Investigators enrolled subjects and allocated them to the VTP and AS groups in a 1:1 ratio using a web-based randomisation system generated by the sponsor and stratified by centre using balanced blocks of varied size ( 2 or 4 subjects). Treatment was open-label (subjects and investigational site staff were not blinded to study treatment), but primary efficacy outcomes were evaluated in a blinded manner.

\section{Procedures}

AS was conducted according to best practice at the time of study design. ${ }^{10,11}$ It comprised a protocol-directed biopsy at 12-month intervals and 3-monthly PSA measurement coupled with a digital rectal exam.

The aim of VTP was to treat a complete prostate lobe. Subjects randomised to padeliporfin VTP underwent pretreatment multiparametric MRI, which was centrally reviewed with the 
biopsy results by a committee composed of radiologists and urologists who made detailed recommendations on the number, length, and position of interstitial optical fibres using treatment guidance software. ${ }^{8,12}$ The treatment-guidance software was used to generate a light-density index (LDI; a measure of the energy exposure per unit volume of target tissue) of $>1$, which had been associated with a high probability of a single-lobe ablation in earlier studies. ${ }^{8}$ However, the urologist in charge of the treatment was allowed to adapt the treatment recommendations to the actual volume and shape of the prostate observed on the TRUS images at the time of the procedure. Once the fibres were accurately positioned in the prostate to cover the desired treatment zone, $4 \mathrm{mg} / \mathrm{kg}$ padeliporfin (Aptuit Glasgow Ltd, Glasgow, UK) was administered intravenously over ten minutes. The drug was activated in the treatment zone by laser light at $753 \mathrm{~nm}$ with a fixed power of $150 \mathrm{~mW} / \mathrm{cm}$ over 22 minutes and 15 seconds, corresponding to an energy dose of $200 \mathrm{~J} / \mathrm{cm} .{ }^{13}$ Subjects with bilateral cancer received a second procedure for contralateral lobe treatment. Retreatment of lobes positive for PC at the Month 12 biopsy was permitted. The VTP procedure was carried out under a general anaesthetic during a 2-hour operating theatre allocation with a planned overnight stay. The urethral catheter was removed the morning after the procedure.

For subjects in both groups, PSA was measured and digital rectal examination performed every three months. TRUS-guided, 12-core biopsy ( 6 cores directed to each prostate lobe) was performed at Months 12 and 24. Thus, the sampling density (number of cores per unit volume of tissue) in the subjects who received VTP was greater than in those in the AS group, particularly for VTP-treated lobe(s) with reduced volume associated with posttreatment fibrosis. Biopsy samples were read centrally by an independent pathologist blinded to treatment assignment and local pathologist reading. An independent, blinded Outcomes Review Panel reviewed all PSA data and TRUS-guided biopsy reports to assess these results and determined the number and location of positive cores. In the case of discrepancy between 
the local and central biopsy readings, the panel's pathologist adjudicated. Any additional radical PC treatments, metastases, evidence of T3 disease, and severe PC-related events were recorded at Months 12 and 24. Any man who underwent radical PC treatment without histological progression (because of patient or physician preference) continued in the study until the end (Month 24) and returned to standard care after that.

The International Prostate Symptom Score (IPSS) and International Index of Erectile Function - 15 Questions (IIEF-15) questionnaires were administered every three months through Month 12 and at Month 24 (and at seven days postprocedure for subjects who received padeliporfin VTP). Validity and sensitivity of these questionnaires to detect change in genitourinary function have been established. ${ }^{14,15}$ The EuroQol-5D (EQ-5D) questionnaire was administered at Month 12 and Month 24 to assess quality of life. All adverse events (AEs) were recorded from the signing of the consent form through the end of the study (including any occurring after the initiation of additional PC treatment). At each study visit, the investigator questioned the subject about AEs and intercurrent illnesses since his last visit. The questions were general, and the presence or absence of specific AEs was not solicited from subjects. AE severity was graded according to the National Cancer Institute Common Terminology Criteria for Adverse Events version 4.03. The investigator assessed the relationship of each $\mathrm{AE}$ to the study drug (padeliporfin), device, and procedure. AEs were coded and categorised according to the Medical Dictionary for Regulatory Activities (version: 18.0). Haematology, coagulation, serum chemistry, and urinalysis were evaluated every three months. Troponin was measured before discharge and quantitative D-Dimer before anaesthesia, before discharge, and at 7 days post-treatment for subjects who received VTP. Vital signs, electrocardiogram, and physical examination were performed preprocedure and postprocedure for subjects who received padeliporfin VTP. An independent Data Safety Monitoring Board (composed of two urologists, a laser surgery expert, and a statistician) 
reviewed safety data and SAE reports throughout the study and advised the sponsor on matters of subject safety.

\section{Outcomes}

The prespecified co-primary efficacy endpoints were treatment failure (histological progression of cancer from low to moderate or higher risk over 24 months follow-up) and absence of definitive cancer (absence of any histology result definitively positive for cancer at Month 24). Moderate or higher risk was defined as the observation of one of the following events: more than three cores definitively positive for cancer when considering all histological results available during follow-up in the study, any Gleason primary or secondary pattern of 4 or more, at least one cancer core length $>5 \mathrm{~mm}, \mathrm{PSA}>10 \mathrm{ng} / \mathrm{mL}$ in three consecutive measures, any T3 PC, metastasis, PC-related death. The prespecified secondary objective was to determine any differences between the two groups in the following outcomes: total cancer burden in the prostate; rate of additional PC radical therapy; rate of severe PC-related events (cancer extension to T3, metastasis, PC-related death); rate of AEs; rate of incontinence, erectile dysfunction, and urinary symptoms.

\section{Statistical analysis}

The sample size was based on an expected rate of progression from low to moderate or higher risk of $\geq 15 \%$ over 2 years in the AS group and $5 \%$ in the VTP group. Using these assumptions, the sample size required was 400 subjects (200 subjects per group), and at least 40 events (subjects with progression of cancer) needed to be observed for the final analysis to take place.

Statistical analyses were conducted using SAS version 9.3. All randomised subjects were analysed for efficacy according to assigned treatment in an intention-to-treat analysis. 
302 Treatment failure (progression) was analysed by survival analysis. Times to progression were compared between the two treatment groups using the log-rank test and quantified using a

Cox proportional-hazards regression model to derive hazard ratios at Month 24, and treatment group and age, number of positive cores, prostate volume, and disease status at baseline were used as covariates. Absence of definitive cancer (positive biopsy) was analysed as a dichotomous outcome. Proportions of subjects with observed success at Month 24 were compared by 2-sided Pearson's chi-square test, and odds and risk ratios were calculated.

Time to initiation of radical therapy was estimated by the Kaplan-Meier method, and the logrank test was used for comparison. The mean number of positive cores and maximum cancer core length at Months 12 and 24 were compared by Student $t$ test. Other efficacy data were summarised descriptively.

All subjects randomised to VTP who received any padeliporfin or initiated any study treatment-related procedure and all subjects randomised to AS were analysed for safety by treatment received. IIEF-15, IPSS, and EQ-5D results were analysed by analysis of covariance. Other safety data, including, AEs, were summarised descriptively.

The trial is registered at ClinicalTrials.gov (NCT01310894).

\section{Role of the funding source}

The study sponsor and funder, STEBA Biotech S.A., developed the protocol in consultation with the study investigators and the European Medicines Agency (EMA). STEBA performed data management and statistical analysis and provided medical writing support for this report. AAzzouzi and ME had full access to all data in the study. The final decision to submit this report for publication was made jointly by all the authors. The corresponding author (ME) had the final responsibility to submit for publication. 


\section{RESULTS}

326 Subjects were recruited from March 8, 2011 to April 30, 2013 and followed for approximately 24 months at 47 university centres at community hospitals in ten European countries (Belgium, Finland, France, Germany, Italy, Netherlands, Spain, Sweden, Switzerland, and the United Kingdom). Tables showing investigational sites, principal investigators, and numbers enrolled at each site and in each country are included in the Appendix (pp.1-2). The study was completed on June 25, 2015, and atotal of 413 men were enrolled: 206 randomised to the VTP group and 207 to the AS group. More subjects in the AS group ( $n=6)$ than in the VTP group $(n=17)$ withdrew consent before study completion. Although unwillingness to accept randomisation to either group was an exclusion criterion, the sponsor anticipated that subjects randomised to AS might withdraw because they had the two groups (Figure 1).

Demographic and baseline disease characteristics were well balanced between the two groups and fit the profile of low-risk PC patients (Table 1). Of the 206 subjects randomised to VTP, nine did not subsequently start the VTP procedure: three who withdrew consent, three who were excluded because for exclusion criteria (bladder cancer discovered on pretreatment MRI, previous Gleason 3+4 biopsy, history of transurethral prostate resection), one who was discontinued by the investigator because of noncompliance, one who had a myocardial infarction, and one who was claustrophobic so unable to undergo the pretreatment MRI.

Of the 197 subjects who started the VTP procedure, one had an anaesthesia reaction before receipt of any padeliporfin or laser treatment. In all, 196 subjects received initial VTP (Figure 1). Of these, 62 received subsequent contralateral treatment, 11 received retreatment, and two 
received both contralateral treatment and retreatment. An LDI $\geq 1$ was achieved in $252(98 \%)$ of 254 initial treatments of a lobe. Prostate lobes that were retreated were less likely to achieve an LDI $\geq 1$, although they were exposed to the same energy of $200 \mathrm{~J} / \mathrm{cm}$ (appendix p.2).

All 413 randomised subjects were included in the efficacy analysis (Figure 1). Padeliporfin VTP delayed progression from low-risk to moderate or higher-risk PC and reduced the probability of a positive biopsy results at Month 24 compared to AS (Table 2). Padeliporfin VTP doubled time to progression from $14 \cdot 1(95 \%$ CI: 12.9 to 23.8$])$ months to $28 \cdot 3$ (95\% CI: 26.0 to 30.6$)$ months $(\mathrm{p}<0 \cdot 0001)$. The rate of progression over 24 months was reduced to approximately one-third that of AS (adjusted hazard ratio $=0 \cdot 34 ; 95 \%$ CI: 0 24, 0.46; $\mathrm{p}<0 \cdot 0001)$. The distribution of predefined progression criteria showed that padeliporfin VTP was efficacious against the individual parameters of the composite progression endpoint. The principal determinants of progression were Gleason grade $\geq 4$ and increases in number of positive cores and cancer core length, which all showed substantial reduction in the padeliporfin VTP group. The regression coefficients showed no effect of treatment group or baseline characteristics. Padeliporfin VTP also increased the probability of a negative Month 24 biopsy by from $13 \cdot 5 \%$ ( 28 of 207 subjects) to $49 \cdot 0 \%$ (101 of 206 subjects) (adjusted risk ratio: $3 \cdot 67 ; 95 \%$ CI: $2 \cdot 53,5 \cdot 33)$; p <0.0001). Eight subjects experienced a severe PC-related event within 24 months, but only one of the subjects who did have such an event (both T3 PC and metastasis) was in the VTP group. This subject was probably under-staged at study entry. His first protocol-required biopsy resulted in a Gleason upgrading that, for the purposes of the study, constituted his first—and therefore reported — progression event. Subsequent investigation revealed a locally advanced PC, and metastasis was detected on further staging investigation. VTP exposure was associated with a reduction in the rate of radical therapy compared to men allocated to AS (12 [5.8\%] of 206 subjects versus 60 [29.0\%] of 207 
subjects; $p<0 \cdot 0001)$ and in time to radical therapy $(\mathrm{p}<0 \cdot 0001)$ (Figure 2$)$. For subjects whose PC did not progress during the study, padeliporfin VTP also produced clinically and statistically significant decrease compared to AS at Month 24 in all mean tumour burden parameters: total number of positive cores ( 0.9 vs 2.3 ; $p<0 \cdot 0001)$, total cancer core length (2.6 vs $6.8 \mathrm{~mm} ; \mathrm{p}<0 \cdot 0001)$, and maximum cancer core length (1.6 vs $3.4 \mathrm{~mm} ; \mathrm{p}<0 \cdot 0001)$. Moreover, VTP produced a stable reduction in PSA of about $3 \mathrm{ng} / \mathrm{mL}$ over the course of the study.

The nine subjects randomised to VTP but who had no treatment-related procedure were excluded from the safety analysis (Figure 1). In the VTP group, IIEF-15 and IPSS assessments showed transient deterioration in erectile and urinary function, but the Month 24 result was comparable between the two groups (appendix p.3). Month 24 IPSS was 6.6 (standard deviation [SD]: 5.47) for VTP and 8.2 (SD: 6.47) for AS, and Month 24 IIEF-15 was 15 (SD: 10.70) for VTP and 16.8 (SD: 11.17) for AS. These results show no significant effect of padeliporfin VTP on genitourinary function compared to AS. The mean EQ-5D questionnaire scores at Month 24 in both the VTP and AS groups were slightly decreased from baseline with no difference in the two groups (82.5 [SD: 12.31] in the VTP group and 81.8 [SD: 12.09] in the AS group), indicating no decrease in quality of life associated with VTP at Month 24 (appendix p.2).

As expected, both the incidence and severity of AEs and SAEs were higher in the VTP group than in the AS group (Table 3). Most subjects in the VTP group experienced an AE, most of which were mild or moderate in severity and self-limited. The most commonly reported AEs in the padeliporfin VTP group were urinary tract infections (23 AEs in 21 [10.7\%] subjects) and AEs in the renal and urinary disorders (280 AEs in 133 [67.5\%] of 197 subjects) and reproductive system and breast disorders system organ classes (197 AEs in 121 [61.4\%] of 197 subjects), and these AEs accounted for the largest differences between the treatment 
groups. AEs related to the study drug, device, or procedure were common but generally not severe. Most of these related AEs occurred during the procedure or in the days immediately after the procedure and resolved quickly without sequelae. The reporting of pain that was thought to be related to the procedure (due to the transcutaneous needle placement, due to the swelling of the prostate or both) was captured by the term 'perineal pain'. This was reported by $30(15 \%)$ men allocated to the VTP group and by $1(0.5 \%)$ man in the AS group.

Three subjects experienced events that were more long-lasting: two with urethral strictures requiring endoscopic dilation and one case of urinary incontinence in a subject who had previously undergone transurethral prostatectomy (TURP). Men with a history of surgery for benign prostatic hypertrophy (including TURP) were subsequently excluded from the study (via protocol amendment 23 October 2012) for safety reasons. All other reports of incontinence were self-limited, were usually urge-related and occurred in the period after catheter withdrawal. Incontinence management was at the discretion of the investigator. The most common related SAE in the VTP group was urinary retention. Typically this event occurred on the first attempt to withdraw the urinary catheter (day-1 post-op). This was managed with immediate recatheterization. The timing of a second attempt at removal of the urinary catheter was left to the discretion of the local investigator. All 15 retention cases resolved within two months. No subject discontinued VTP because of an AE. Three subjects discontinued the study because of AEs. One subject in the AS group developed ureteric cancer. One subject in the VTP group had an anaphylactic reaction to the anaesthesia administered at the start of the VTP procedure; he had received no padeliporfin or VTP. One subject in the VTP group died from a myocardial infarction during mountain climbing approximately eight months after padeliporfin VTP, and the investigator assessed the AE as unrelated to study drug, device, or procedure. 
423 An independent Data and Safety Monitoring Board reviewed safety data approximately every

4243 months throughout the study and advised the study sponsor on matters of subject safety. At

425 all meetings, the members unanimously agreed that no safety issues had emerged in the 426 study.

\section{DISCUSSION}

VTP doubled time to progression (from $14 \cdot 1$ to $28 \cdot 3$ months), reduced the progression rate to approximately one-third that of AS, and increased the probability of a negative prostate biopsy at 24 months post-treatment from $13 \cdot 5 \%$ to $49 \cdot 0 \%$. VTP was also safe and well tolerated with only minor and transient deterioration in genitourinary function. Our study has shown that partial-gland ablation by VTP influences the course of PC in the short-to-medium term. First, the proportion of men who transition from a cancer status to cancer-free status was increased. Second, the proportion of men who progress from a histologically defined low-risk status to a higher one is diminished. As a result, fewer men chose to undergo radical therapy during the study period. Moreover, these benefits were achieved safely, efficiently, and without compromising genitourinary function when assessed at 12 and 24 months after VTP.

Since this is the first comparative efficacy study of its type, it is important to consider the methodological considerations that were inherent in its design and conduct. The first relates to the population studied. By today's standards this population might be considered low risk.

443 However, whilst the study was in development and being discussed with the EMA, neither 444 AS nor focal therapy were accepted as standard care. The EMA agreed that we could reasonably exclude very-low-risk patients. Therefore, lower and upper thresholds of risk (defined by Gleason pattern and tumour burden) were set, below which and above which men 
were excluded. This low-risk group was the only one that could have been studied at the time.

448 Were the study designed today, given the changes to risk categorisation, it is likely that men with well characterised PC and low volume secondary Gleason pattern 4 would be included. ${ }^{16}$

450

451

452

453

454

455

456

457

458

459

460

461

462

A second limitation relates to rapidly changing practice in risk stratification of PC patients, most significantly the use of MRI in the diagnostic and the re-evaluation phases of both AS and focal therapy. ${ }^{17,18}$ When the study began, few units offered MRI to patients on AS or as part of the work-up for focal therapy. Now it is difficult to imagine using either strategy without MRI. Although only men assigned to VTP had MRI in this study, images were used only for treatment planning, not for detection or staging. The only way in which unilateral use of MRI could have biased subjects' allocation was the detection of colorectal or bladder cancer, which would have triggered a study withdrawal. If the study were repeated today, MRI would play an important role in subject selection and risk stratification for both interventions. ${ }^{19}$

A third concern is discriminating true progression from reclassification. When using a biopsy-based strategy to refine the risk stratification at given intervals in AS, upgrading (transition from an exclusive Gleason pattern 3 status to one with elements of Gleason pattern 4 or 5) occurs. Determining whether the observed increase in the Gleason pattern is a correction of inherent diagnostic imprecision or the product of true disease progression has proved challenging. Whilst no universal definition of clinical significance exists, recently published MRI studies have used the presence of Gleason pattern 4 as the minimum definition of clinically significant PC. ${ }^{17,18}$ Physicians have recommended treatment upon upgrading irrespective of its underlying cause. This strategy appears prudent given that recently published data from two mature AS series have identified higher risk groups (within the risk profile suitable for AS) that are at greater risk of progression. ${ }^{2,20,21}$ 
471 The final issue relates to the efficacy endpoints evaluated. If endpoints such as progression to 472 metastases or death had been used, the natural history of low-risk PC would have required a very large study conducted over two decades. Some experts advocate prioritizing of shorterterm, relevant outcomes that are important to patients to support patients and their physicians in their clinical decision-making. ${ }^{22}$

This multicentre study has demonstrated that padeliporfin VTP can be implemented widely and delivered effectively and safely. The latter issue deserves some qualification. Exposure to VTP resulted in an increase in the frequency of SAEs from 1 in 10 men on AS to 1 in 3 men who received VTP. Most of the events were expected, genitourinary in nature, and selflimited. The most important of these events was failure to void on catheter removal (urinary retention). The event was managed by replacement of the urethral catheter and extension of the period of dependent urinary drainage.

It is worth noting that most study sites had no prior experience in delivering focal therapy, let alone VTP. Study recruitment was timely over a large geographical area, a scenario that contrasts with the many previous attempts to undertake randomised, comparative studies of early PC treatment, which either failed to recruit completely or closed because of poor recruitment. ${ }^{23}$ Feasibility is an important attribute for surgical interventions, and our results demonstrate that VTP can be taught, learned, and delivered by a range of health care providers and systems. This study was performed at a large number of centres and in a variety of healthcare systems, few of which had any previous experience with VTP, and yet we managed to achieve a very low rate of permanent urinary toxicity. Since our understanding and management of early PC have changed so much in the last few years, it is worth speculating on how padeliporfin VTP might be used with current diagnostics and risk stratification, which are unrecognizable from those at the time of study design. Adoption of MRI and targeted biopsy into the clinical pathway has created more precise risk stratification, 
allowing a more nuanced approach to men with a new PC diagnosis. Given that MRI is now

widely used within the diagnostic pathway but was not used for diagnosis or risk stratification in our study, it is worth speculating on how the diagnostic process may be influenced by the results of this study. First, it is likely that a pathway based on MRI—-because of its role as a triage test between an elevated PSA and biopsy—will result in a reduction of the number of men biopsied and in the proportion of men receiving the diagnosis of clinically insignificant PC. In contrast, men with an MRI abnormality will undergo targeted biopsy (something that was not possible without MRI), resulting in a greater sensitivity for clinically significant disease. It is very likely that the men with clinically significant isolated lesions will be the candidates for focal prostate therapy. Men who do not need treatment should not have it. Men who require whole-gland treatment because of bilateral clinically significant disease should be offered it. Men with locally advanced disease should be offered multimodality therapy. However, men who have low-risk, localised disease can now choose, on the basis of the evidence that our study has generated, how to approach tissue preservation.

More research is needed to address unanswered questions, the principal one being the longterm effect of tissue-preserving treatment on PC control rates. One unknown element is the

512 efficacy of padeliporfin VTP in eradicating cancers of different grades within the target volume. A study in men with Gleason pattern 4 (NCT01875393) has been submitted for publication. Another uncertainty relates to the stability of the tissue that lies beyond the treatment zone. This question requires long-term follow-up, which has been initiated in the men from Study CLIN1001 PCM301.

\section{CONTRIBUTORS}

518 The sponsor, STEBA Biotech S.A., developed the study design in consultation with the study investigators and the EMA. BA performed the statistical analysis and interpretation on behalf 
520 of the sponsor. AAzzouzi, SV, EB, AC, FK, HVP, CGS, JR, GS, ES, AAlcaraz, TTT, DJR,

521 FGV, GA, ME, and the PCM301 Study Group conducted the study and collected the data.

522 FMJD chaired the Data Safety Monitoring Board and GF and CG served on the Outcomes

523 Review Panel. ME prepared the first draft of the manuscript and with Anne McDonough, a

524 professional medical writer funded by the sponsor. All authors contributed to the final data

525 interpretation and final draft of the report and approved submission for publication.

526 
AAzzouzi, SV, EB, AC, FK, HGV, CGS, JR, GS, ES, AAlcaraz, TTT, DJR, FGV, GA, and ME received payment from STEBA as investigators on this study. AA and ME have also acted as consultants and proctors for STEBA. FMJD, GF, and CG received payment from STEBA for other roles on the study (Data Safety Monitoring Board, Outcomes Review Panel). BA is a statistical consultant to STEBA. FB and BG are employees of STEBA. FGV reports receipt of funding for research from Astellas Pharma and acting as a paid proctor for

534 Intuitive Surgical, Inc. AAlcaraz reports payment for speaking engagements from several companies (Astellas Pharma, Janssen Pharmaceutica, Sanofi, Bayer, STEBA Biotech S.A.,

Olympus Corporation). TTT reports being an advisor for Astellas Pharma, Ferring

Pharmaceuticals, Orion Corporation, and Bayer and receiving institutional funding from

Astellas Pharma, Ferring Pharmaceuticals, Medivation, Inc, Orion Corporation, and Bayer.

ME reports acting as a principal/co-investigator in a number of PC studies supported by

SonaCare Medical, Sophiris Bio Inc, and TROD Medical and as a consultant/advisor to GSK and Sanofi-Aventis, being a founding partner of London Urology Associates, and shareholdings in Nuada Medical Ltd.

\section{ACKNOWLEDGEMENTS}

544 We thank the patients who agreed to participate in this study.

545 We thank Drs. Peter Scardino and Michael Zelefsky (Memorial Sloane Kettering Cancer

546 Center, New York, USA) for their very helpful comments and advice on earlier versions of 547 this manuscript. 

Investigator. His research is supported by the UCLH/UCL NIHR Biomedical Research

\section{REFERENCES}

552

1. Mottet N, Bellmunt JE, van den Bergh RCN, et al. Guidelines on Prostate Cancer. European Association of Urology; 2015. http://uroweb.org/wp-content/uploads/EAUGuidelines-Prostate-Cancer-2015-v2.pdf. Accessed 9 June 2016.

2. Klotz L, Vesprini D, Sethukavalan P, et al. Long-term follow-up of a large active surveillance cohort of patients with prostate cancer. J Clin Oncol. 2015; 33: 272-7.

3. Klotz L, Emberton M. Management of low risk prostate cancer: active surveillance and focal therapy. Curr Opin Urol. 2014; 24: 270-9.

4. van den Bergh RC, Ahmed HU, Bangma CH, Cooperberg MR, Villers A, Parker CC.. Novel tools to improve patient selection and monitoring on active surveillance for low-risk prostate cancer: a systematic review. Eur Urol. 2014; 65: 1023-31.

5. Ahmed HU, Hindley RG, Dickinson L, et al. Focal therapy for localised unifocal and multifocal prostate cancer: a prospective development study. Lancet Oncol. 2012; 13: 622-32.

6. Valerio M, Ahmed HU, Emberton M, et al. The role of focal therapy in the management of localised prostate cancer: a systematic review. Eur Urol. 2014; 66: 732-51.

7. Azzouzi AR, Barret E, Bennet J, et al. TOOKAD® soluble focal therapy: pooled analysis of three phase II studies assessing the minimally invasive ablation of localized prostate cancer. World J Urol. 2015; 33: 945-53.

8. Azzouzi AR, Barret E, Moore CM, et al. TOOKAD(®) Soluble vascular-targeted photodynamic (VTP) therapy: determination of optimal treatment conditions and 
assessment of effects in patients with localised prostate cancer. BJU Int. 2013; 112:

574

575

576

577

578

579

580

581

582

583

584

585

586

587

588

589

590

591

592

593

594

595

596

597 766-74.

9. Welty CJ, Cowan JE, Nguyen H, et al. Extended followup and risk factors for disease reclassification in a large active surveillance cohort for localized prostate cancer. $J$ Urol. 2015;193: 807-11.

10. Heidenreich A, Bellmunt J, Bolla M, et al. EAU guidelines on prostate cancer. Part 1: screening, diagnosis, and treatment of clinically localised disease. Eur Urol. 2011; 59: $61-71$.

11. American Urological Association. Guideline for the Management of Clinically Localized Prostate Cancer: 2007 Update.

http://www.auanet.org/common/pdf/education/clinical-guidance/Prostate-Cancer.pdf. Accessed 9 June 2016.

12. Moore CM, Azzouzi AR, Barret E, et al. Determination of optimal drug dose and light dose index to achieve minimally invasive focal ablation of localised prostate cancer using WST11-vascular-targeted photodynamic (VTP) therapy. BJU Int. 2015; 116: 888-96.

13. Azzouzi AR, Lebdai S, Benzaghou F, Stief C. Vascular-targeted photodynamic therapy with TOOKAD® Soluble in localized prostate cancer: standardization of the procedure. World J Urol. 2015; 33: 937-44.

14. Barry MJ, Fowler FJ Jr, O'Leary MP, et al. The American Urological Association symptom index for benign prostatic hyperplasia. The Measurement Committee of the American Urological Association. J Urol. 1992; 148: 1549-57.

15. Rosen RC, Riley A, Wagner G, Osterloh IH, Kirkpatrick J, Mishra A. The international index of erectile function (IIEF): a multidimensional scale for assessment of erectile dysfunction. Urology 1997; 49: 822-30. 
16. Epstein JI. A new contemporary prostate cancer grading system. Ann Pathol. 2015; 35: 474-6.

17. Panebianco V, Barchetti F, Sciarra A, et al. Multiparametric magnetic resonance imaging vs. standard care in men being evaluated for prostate cancer: a randomized study. Urol Oncol. 2015; 33: 17.e1-7.

18. Recabal P, Assel M, Sjoberg DD, et al. The efficacy of multiparametric magnetic resonance imaging and magnetic resonance imaging targeted biopsy in risk classification for patients with prostate cancer on active surveillance. J Urol. 2016; 196: $374-81$.

19. Jarow JP, Ahmed HU, Choyke PL, Taneja SS, Scardino PT. Partial gland ablation for prostate cancer: report of a Food and Drug Administration, American Urological Association, and Society of Urologic Oncology Public Workshop. Urology 2016; 88: $8-13$.

20. Henderson DR, de Souza NM, Thomas K, et al. Nine-year follow-up for a study of diffusion-weighted magnetic resonance imaging in a prospective prostate cancer active surveillance cohort. Eur Urol. 2015; pii: S0302-2838(15)00980-X. doi: 10.1016/j.eururo.2015.10.010. [Epub ahead of print].

21. Godtman RA, Holmberg E, Khatami A, Pihl CG, Stranne J, Hugosson J. Long-term results of active surveillance in the Göteborg randomized, population-based prostate cancer screening trial. Eur Urol. 2016; pii: S0302-2838(16)30026-4. doi: 10.1016/j.eururo.2016.03.048. [Epub ahead of print].

22. D'Amico AV. Personalizing the use of active surveillance as an initial approach for men with newly diagnosed prostate cancer. J Clin Oncol. 2015; 33: 3365-6.

23. Ahmed HU, Berge V, Bottomley D, et al. Can we deliver randomized trials of focal therapy in prostate cancer? Nat Rev Clin Oncol. 2014; 11: 482-91. 
624 Table 1. Demographic and baseline prostate cancer characteristics

\begin{tabular}{|c|c|c|c|}
\hline Characteristic & $\begin{array}{c}\text { Padeliporfin VTP } \\
\quad \mathrm{N}=\mathbf{2 0 6}\end{array}$ & $\begin{array}{l}\text { Active surveillance } \\
\qquad \mathbf{N}=\mathbf{2 0 7}\end{array}$ & $\begin{array}{c}\text { Total } \\
\mathrm{N}=\mathbf{4 1 3}\end{array}$ \\
\hline \multicolumn{4}{|l|}{ Age (years) } \\
\hline Mean (SD) & $64.2(6.7)$ & $62.9(6.7)$ & $63.5(6.7)$ \\
\hline Range: minimum, maximum & 45,85 & 44,79 & 44,85 \\
\hline \multicolumn{4}{|l|}{ Race } \\
\hline Caucasian, n (\%) & $202(98.1)$ & $206(99.5)$ & $408(98.8)$ \\
\hline Other, $\mathrm{n}(\%)$ & $4(2.9)$ & $1(0.5)$ & $5(1.2)$ \\
\hline \multicolumn{4}{|l|}{ Body mass index $\left(\mathrm{kg} / \mathrm{m}^{2}\right)$} \\
\hline Mean (SD) & $26.5(3.3)$ & $27.3(4.0)$ & $26.9(3.7)$ \\
\hline Range: minimum, maximum & $18.8,38.6$ & $18.8,44.8$ & $18.8,44.8$ \\
\hline \multicolumn{4}{|l|}{ Time since diagnosis (months) } \\
\hline Mean (SD) & $6.3(8.5)$ & $6.0(7.9)$ & $6.2(8.2)$ \\
\hline Range: minimum, maximum & $0.2,54.2$ & $0.2,47.4$ & $0.2,54.2$ \\
\hline \multicolumn{4}{|l|}{ TNM staging } \\
\hline T1a, n (\%) & $1(0.5)$ & 0 & $1(0.2)$ \\
\hline T1c, n (\%) & $177(85.9)$ & $180(87.0)$ & $357(86.4)$ \\
\hline $\mathrm{T} 2 \mathrm{a}, \mathrm{n}(\%)$ & $28(13.6)$ & $27(13.0)$ & $55(13.3)$ \\
\hline \multicolumn{4}{|l|}{ PSA (ng/mL) } \\
\hline Mean (SD) & $6.2(2.1)$ & $5.9(2.0)$ & $6.1(2.1)$ \\
\hline Range: minimum, maximum & $0.1,10.0$ & $0.5,10.0$ & $0.1,10.0$ \\
\hline \multicolumn{4}{|l|}{ Estimated prostate volume (cc) } \\
\hline Mean (SD) & $42.5(12.5)$ & $42.5(11.8)$ & $42.5(12.1)$ \\
\hline Unilateral disease, $\mathrm{n}(\%)$ & $157(76.2)$ & $163(78.7)$ & $320(77.5)$ \\
\hline Bilateral disease, $\mathrm{n}(\%)$ & $49(23.8)$ & $44(21.3)$ & $93(22.5)$ \\
\hline \multicolumn{4}{|c|}{ Total number of pretreatment biopsy cores } \\
\hline Mean (SD) & $13.6(3.3)$ & $13.6(3.6)$ & $13.6(3.4)$ \\
\hline Range: minimum, maximum & 10,25 & 10,26 & 10,26 \\
\hline \multicolumn{4}{|c|}{$\begin{array}{l}\text { Total number of pretreatment biopsy cores } \\
\text { with cancer }\end{array}$} \\
\hline Mean $(\mathrm{SD})$ & $2.1(0.7)$ & $2.0(0.7)$ & $2.1(0.7)$ \\
\hline Range: minimum, maximum & 1,3 & 1,3 & 1,3 \\
\hline 1 core, $\mathrm{n}(\%)$ & $39(18.9)$ & $52(25.1)$ & $91(22.0)$ \\
\hline 2 cores, $\mathrm{n}(\%)$ & $110(53.4)$ & $100(48.3)$ & $210(50.8)$ \\
\hline 3 cores, $\mathrm{n}(\%)$ & $57(27.7)$ & $55(26.6)$ & $112(27.1)$ \\
\hline \multicolumn{4}{|l|}{ Total cancer core length $(\mathrm{mm})$} \\
\hline Mean (SD) & $4.3(2.3)$ & $3.8(2.4)$ & $4.1(2.4)$ \\
\hline Range: minimum, maximum & $0^{\mathrm{a}, 14}$ & $0^{\mathrm{a}}, 11$ & 0,14 \\
\hline
\end{tabular}




\section{Table 2. Co-primary efficacy endpoints}

\begin{tabular}{|c|c|c|c|c|}
\hline \multirow[b]{2}{*}{ Endpoint ${ }^{\mathrm{a}}$} & \multirow{2}{*}{$\begin{array}{c}\text { Padeliporfin VTP } \\
\text { N = 206 } \\
\text { n }(\%)\end{array}$} & \multirow{2}{*}{$\begin{array}{c}\text { Active surveillance } \\
\mathbf{N}=\mathbf{2 0 7} \\
\mathbf{n}(\boldsymbol{\%})\end{array}$} & \multicolumn{2}{|c|}{ Padeliporfin VTP vs active surveillance } \\
\hline & & & Ratio (95\% CI) & p value \\
\hline Progression & $58(28.2)$ & $120(58.0)$ & $\begin{array}{c}\text { Adjusted }^{\mathrm{b}} \text { hazard ratio } \\
0.34(0.24,0.46) \\
\end{array}$ & $<0 \cdot 001^{\mathrm{c}}$ \\
\hline \multicolumn{5}{|l|}{ Criteria for progression $^{\mathrm{d}}$} \\
\hline More than three cores positive & $23(11.2)$ & $58(28.0)$ & $\mathrm{NC}$ & $<0.001^{\mathrm{e}}$ \\
\hline Gleason $\geq 4$ & $49(23.8)$ & $91(44.0)$ & $\mathrm{NC}$ & $<0.001^{\mathrm{e}}$ \\
\hline Cancer core length $>5 \mathrm{~mm}$ & $25(12.1)$ & $51(24.6)$ & $\mathrm{NC}$ & $0 \cdot 001^{\mathrm{e}}$ \\
\hline $\begin{array}{l}\text { PSA }>10 \mathrm{ng} / \mathrm{mL} \text { in three consecutive } \\
\text { measures }\end{array}$ & $3(1.5)$ & $14(6.8)$ & $\mathrm{NC}$ & $0 \cdot 007^{\mathrm{e}}$ \\
\hline Any T3 prostate cancer & 0 & $4(1.9)$ & $\mathrm{NC}$ & NA \\
\hline Metastasis & 0 & 0 & $\mathrm{NC}$ & NA \\
\hline Prostate cancer-related death & 0 & 0 & $\mathrm{NC}$ & NA \\
\hline Negative Biopsy at Month 24 & $101(49.0)$ & $28(13.5)$ & $\begin{array}{c}\text { Adjusted }^{\mathrm{f}} \text { risk ratio } \\
3.67(2.53,5.33) \\
\end{array}$ & $<0.001^{\mathrm{e}}$ \\
\hline \multicolumn{5}{|c|}{$\begin{array}{l}\mathrm{CI}=\text { confidence interval; } \mathrm{NA}=\text { not applicable; } \mathrm{NC}=\text { not calculated; } \mathrm{VTP}=\text { vascular-targeted photodynamic therapy. } \\
\text { a The Hochberg procedure was used to adjust for multiplicity of the two co-primary endpoints. } \\
\text { b Cox proportional-hazards model with treatment as fixed effect and baseline age, number of cores positive, prostate volume, and disease status (unilateral/bilateral } \\
\text { as covariates. } \\
\text { c From the log-rank test of equality of survival curves across treatment groups Cox proportional-hazards model with treatment as fixed effect and baseline age, } \\
\text { number of cores positive, prostate volume, and disease status (unilateral/bilateral as covariates. } \\
\text { d A subject might have met > } 1 \text { criterion for progression. } \\
\text { e From Pearson's chi-square test for observed success. } \\
\text { f Logistic regression model with treatment as fixed effect and baseline age, number of cores positive, prostate volume, and disease status (unilateral/bilateral) as } \\
\text { covariates. }\end{array}$} \\
\hline
\end{tabular}


Table 3. Adverse events

\begin{tabular}{|c|c|c|c|c|}
\hline \multirow[b]{2}{*}{ AE category } & \multicolumn{2}{|c|}{$\begin{array}{c}\text { Padeliporfin VTP } \\
\mathbf{N}=197^{*} \\
\end{array}$} & \multicolumn{2}{|c|}{$\begin{array}{c}\text { Active surveillance } \\
\mathbf{N}=\mathbf{2 0 7} \\
\end{array}$} \\
\hline & $\begin{array}{c}\text { Subjects } \\
\text { n (\%) }\end{array}$ & $\begin{array}{c}\text { Events } \\
\text { n }\end{array}$ & $\begin{array}{l}\text { Subjects } \\
\text { n (\%) }\end{array}$ & $\begin{array}{c}\text { Events } \\
\text { n }\end{array}$ \\
\hline All AEs & $187(94.9)$ & 939 & $114(55.1)$ & 307 \\
\hline Drug, device, or VTP procedure-related AE & $155(78.7)$ & 551 & NA & NA \\
\hline All SAEs & $60(30.5)$ & 88 & $21(10.1)$ & 25 \\
\hline Drug, device, or VTP procedure-related SAE & $30(15.2)$ & 39 & NA & NA \\
\hline AE leading to study discontinuation & $2(1.0)$ & 2 & $1(0.5)$ & 1 \\
\hline AE leading to death & $1(0.5)$ & 1 & 0 & 0 \\
\hline \multicolumn{5}{|c|}{ Adverse Events Occurring in $\geq 10 \%$ of Subjects in Either Group } \\
\hline $\begin{array}{l}\text { System Organ Class } \\
\text { Preferred Term }\end{array}$ & \multicolumn{2}{|c|}{$\begin{array}{c}\text { Subjects } \\
\text { n }(\%)\end{array}$} & \multicolumn{2}{|c|}{$\begin{array}{c}\text { Subjects } \\
\text { n }(\%)\end{array}$} \\
\hline \multicolumn{5}{|l|}{ Infections and infestations } \\
\hline Urinary tract infection & \multicolumn{2}{|c|}{$21(10.7)$} & \multicolumn{2}{|c|}{$9(4.3)$} \\
\hline \multicolumn{5}{|l|}{ Renal and urinary disorders } \\
\hline Dysuria & \multicolumn{2}{|c|}{$54(27.4)$} & \multicolumn{2}{|c|}{$5(2.4)$} \\
\hline Haematuria & \multicolumn{2}{|c|}{$56(28.4)$} & \multicolumn{2}{|c|}{$6(2.9)$} \\
\hline Micturition urgency & & & & \\
\hline Pollakiuria & & & & \\
\hline Urinary retention & & & & \\
\hline Reproductive system and breast disorders & & & & \\
\hline Erectile dysfunction & & & & \\
\hline Perineal pain & & & & \\
\hline & by Severity & & & \\
\hline $\begin{array}{l}\text { AE Grade } \\
\text { System Organ Class } \\
\text { Preferred Term }\end{array}$ & & & & \\
\hline Grade 1 (mild) & & & & \\
\hline Grade 2 (moderate) & & & & \\
\hline Grade 3 (severe) & & & & \\
\hline Blood and lymphatic disorders & & & & \\
\hline Thrombocytopenia & & & & \\
\hline Cardiac disorders & & & & \\
\hline Atrial fibrillation & & & & \\
\hline Myocardial infarction & & & & \\
\hline Endocrine disorders & & & & \\
\hline Hypothyroidism & & & & \\
\hline Gastrointestinal disorders & & & & \\
\hline Abdominal pain & & & & \\
\hline Gastrointestinal haemorrhage & & & & \\
\hline Inguinal hernia & & & & \\
\hline Rectal haemorrhage & & & & \\
\hline General disorders and administration site & & & & \\
\hline Device failure & & & & \\
\hline Pyrexia & & & & \\
\hline Immune system disorders & & & & \\
\hline Drug hypersensitivity & & & & \\
\hline Infections and infestations & & & & \\
\hline Epididymitis & & & & \\
\hline Orchitis & & & & \\
\hline Otitis externa & & & & \\
\hline Staphylococcal infection & & & & \\
\hline Urinary tract infection & & & & \\
\hline Injury, poisoning and procedural complica & & & & \\
\hline Accident & & & & \\
\hline Craniocerebral injury & & & & \\
\hline Procedural pain & & & & \\
\hline Investigations & & & & \\
\hline Fibrin D dimer increased & & & & \\
\hline Musculoskeletal and connective tissue diso & & & & \\
\hline Arthralgia & & & & \\
\hline Osteoarthritis & & & & \\
\hline Neoplasms benign, malignant and unspecif & & & & \\
\hline Ear neoplasm & & & & \\
\hline Neuroendocrine carcinoma & & & & \\
\hline Prostate cancer & & & & \\
\hline Tongue cancer recurrent & & & & \\
\hline Tonsillar neoplasm & & & & \\
\hline Ureteric cancer metastatic & & & & \\
\hline
\end{tabular}




\begin{tabular}{|c|c|c|}
\hline Ureteric cancer regional & 0 & $1(0.5)$ \\
\hline \multicolumn{3}{|l|}{ Nervous system disorders } \\
\hline Cerebrovascular accident & $1(0.5)$ & 0 \\
\hline Headache & $1(0.5)$ & 0 \\
\hline Transient ischaemic attack & 0 & $1(0.5)$ \\
\hline \multicolumn{3}{|l|}{ Psychiatric disorders } \\
\hline Depression & $1(0.5)$ & $1(0.5)$ \\
\hline \multicolumn{3}{|l|}{ Renal and urinary disorders } \\
\hline Dysuria & $3(1.5)$ & 0 \\
\hline Haematuria & $1(0.5)$ & 0 \\
\hline Urinary incontinence & $2(1.0)$ & $1(0.5)$ \\
\hline Urinary retention & $3(1.5)$ & $1(0.5)$ \\
\hline \multicolumn{3}{|c|}{ Reproductive system and breast disorders } \\
\hline Ejaculation failure & $2(1.0)$ & 0 \\
\hline Erectile dysfunction & $2(1.0)$ & $3(1.4)$ \\
\hline Perineal pain & $1(0.5)$ & 0 \\
\hline Prostatic pain & $1(0.5)$ & 0 \\
\hline Prostatitis & $3(1.5)$ & $1(0.5)$ \\
\hline \multicolumn{3}{|c|}{ Skin and cutaneous tissue disorders } \\
\hline Purpura & $1(0.5)$ & 0 \\
\hline \multicolumn{3}{|c|}{ Surgical and medical procedures } \\
\hline Aortic valve replacement & 0 & $1(0.5)$ \\
\hline Cataract operation & $1(0.5)$ & 0 \\
\hline Facial operation & $1(0.5)$ & 0 \\
\hline Knee arthroplasty & $1(0.5)$ & 0 \\
\hline \multicolumn{3}{|l|}{ Vascular disorders } \\
\hline Phlebitis & 0 & $1(0.5)$ \\
\hline Thrombosis & 0 & $1(0.5)$ \\
\hline Grade 4 (life-threatening) & $3(1.5)$ & $1(0.5)$ \\
\hline \multicolumn{3}{|l|}{ Cardiac disorders } \\
\hline Angina unstable & $1(0.5)$ & 0 \\
\hline Myocardial infarction & 0 & $1(0.5)$ \\
\hline \multicolumn{3}{|l|}{ Immune system disorders } \\
\hline Anaphylactic reaction & $1(0.5)$ & 0 \\
\hline \multicolumn{3}{|c|}{ Respiratory, thoracic and mediastinal disorders } \\
\hline Bronchospasm & $1(0.5)$ & 0 \\
\hline Grade 5 (death) & $1(0.5)$ & $\mathbf{0}$ \\
\hline \multicolumn{3}{|l|}{ Cardiac disorders } \\
\hline Myocardial infarction & $1(0.5)$ & 0 \\
\hline
\end{tabular}




\section{FIGURE LEGENDS (IN-TEXT)}

Figure 1. Disposition of subjects by treatment group

Figure 2. Time to initiation of radical therapy by treatment group - Kaplan-Meier analysis 